\title{
The use of ozonated water and rectal insufflation in patients with intestinal dysbiosis
}

\author{
Fortunato Loprete, ${ }^{1}$ Francesco Vaiano ${ }^{2}$ \\ ${ }^{1}$ Private practitioner, Surgeon, Padova; ${ }^{2}$ Private practitioner, Surgeon, Desio (MB), Italy
}

\begin{abstract}
A functional intestinal Flora, physiologically speaking, is an important indication of a healthy organism; therefore, it is an important ally for the Function of Defense. The study was conducted on an adult population from January 2012 to June 2013. 34 individuals were considered, regardless of age and sex, of which 23 females (67.6\%) and 11 males (32.4\%), aged between 27 and 62 (average: 43.9 years). For the evaluation of abdominal pressure-like pain and discomfort caused by other typical disorders of dysbiosis, a visual-analogue scale was chosen, according to the Scott and Huskisson model.

The results of the study made it possible to verify - first of all - that intestinal dysbiosis is a disease with a higher incidence with respect to what clinical data does not allow to establish on the basis of subjective and objective symptoms.

In conclusion, the study confirmed the validity of the treatment with ozonized water combined with rectal insufflation of oxygen and ozone mixture.
\end{abstract}

\section{Introduction and Definition}

A functional intestinal Flora, physiologically speaking, is an important indication of a healthy organism; in fact, it is established that normal Microflora is not only the first barrier to infections, but it also has other functions that help maintain good human health. ${ }^{1}$

Therefore, it is an important ally for the Function of Defense,

Correspondence: Fortunato Loprete and Francesco Vaiano, OxygenOzone Therapy Scientific Society (SIOOT), via Roma 69, 24020 Gorle (BG), Italy.

E-mail: dottf.loprete@gmail.com ; francescovaiano@fastwebnet.it

Key words: Ozonated water; rectal insufflation; intestinal dysbiosis.

Received for publication: 23 December 2017.

Accepted for publication: 28 December 2017.

CCopyright F. Loprete and F. Vaiano, 2017

Licensee PAGEPress, Italy

Ozone Therapy 2017; 2:7304

doi:10.4081/ozone.2017.7304

This article is distributed under the terms of the Creative Commons Attribution Noncommercial License (by-nc 4.0) which permits any noncommercial use, distribution, and reproduction in any medium, provided the original author(s) and source are credited. antagonistic in regard to pathogenic germs and harmful bacteria thanks to the production of natural antibiotics and bacteriocins. ${ }^{2}$

When physiological Flora is balanced and in symbiosis with the organism it becomes beneficial for both, and is referred to as Eubiosis. $^{3}$

When, for various reasons, which we will get to later, there is an alteration of the microbial ecosystem with a disruption and gradual alteration of the normal microbial flora, then we are dealing with Dysbiosis.

More precisely it is known as intestinal dysbiosis, referring to a set of symptoms and functional gastrointestinal disorders that can evolve into diseases involving organs or systems distant from the colon, through microbial lymphogenous propagation with the blocking of MALT and GALT. ${ }^{4}$

It represents, in fact, a very common social problem, caused by the functional alteration of the colonic mucosa and the modification of the intestinal bacterial flora, with consequent alteration of the composition of the bacterial flora residing in the intestine.

If we consider the vast surface of the gastro-intestinal mucosa and its grand capacity for absorption, we can comprehend that an alteration of intestinal conditions generates many toxic substances (toxins), which, after being circulated through the blood and lymphatic pathways, are distributed in various parts of the body creating multiple dysfunctions and pathologies.

Following are some of the main symptoms of dysbiosis: poor digestion, swelling, constipation alternating with dysentery, fecal mucus, mood changes, sleep disturbances, vaginal candidiasis, dysmenorrhea, asthenia, halitosis, headaches, allergies, etc. ${ }^{5}$

The diagnosis of intestinal dysbiosis is made through:

Medical history: recently taken medication, diet, smoking, work environment, lifestyle etc.

Symptoms: flatulence, constipation and/or diarrhea, chronic gingivitis, meteorism, chronic asthenia, food intolerances up to actual allergies in general, chronic gastritis, headaches, etc.

Laboratory data: alterations of transaminases and alpha-amylases, coproculture, fecal $\mathrm{pH}$ (in the case of normal intestinal flora the $\mathrm{pH}$ of the large intestine corresponds to a value between 5.5 and 6.0 from childhood to adulthood. Values above $\mathrm{pH} 6.0$ are already suspicious); indole test in urine samples (in urine there are normally small amounts of indole equal to $4-20 \mathrm{mg}$ in the 24 hours). In case of severe intestinal dysbiosis, skatole is present in the urine as well.

Regarding the therapeutic approach to the pathology, we must remember that the complexity of the pathogenetic and pathophysiological phenomena that characterize the dysbiotic phenomenon must inevitably provide, from a therapeutic point of view, an integrated intervention that aims, on the one hand, to act on the host and on the other, to the homeostatic rebalancing of the intestinal microclimate, restoring the balance between the different bacterial species. 
Therefore, it is of fundamental importance to restore a correct balance of bacterial flora, through an appropriate combined treatment aimed both at the drainage of the gastrointestinal system and its functionally related organs (liver, pancreas, etc.), and at preparing the colon for the subsequent treatment of recolonization using an oxygen-ozone mixture and probiotics.

Ozone therapy (by means of rectal insufflation and especially by means of hyperozonated water administered orally) has proved to be, in recent years, a medical treatment with vast application possibilities. Its effectiveness has been demonstrated in functional disorders of the colon and in the rehabilitation of the peristaltic intestinal capacity.

Patients tolerate this therapy well and it has no short or longterm side effects. MULTIOSSIGEN ozone therapy by rectal insufflation and hyperozonated water by mouth returns functionality to the intestine with all the powers for a valid defense against bacteria, viruses and any other toxicity and dysfunction.

\section{Purpose of the study}

The goal of the study, which lasted a total of 90 days, was to verify the effects induced by ozonated water and rectal insufflation on the overall well being of 34 individuals affected by intestinal dysbiosis.

The authors show how the mixture of $\mathrm{O}_{2}-\mathrm{O}_{3}$ represents a valid aid for the restoration of the physiological status of the altered mucosa, thus ensuring the digestive and detoxifying functions of the gastrointestinal tract.

At the beginning, after 45 days and at the end of the study, at 90 days, a certain symptoms (considered the most important ones by the Authors) were evaluated in the subjects, typical of the pathology treated (chronic asthenia, meteorism, halitosis, alternate alveolus (constipation alternating with diarrhea), poor digestion, gastralgia, irritable bowel, aerophagia and bothersome eructation).

The diagnosis was made by dosing two metabolites, indican (dosed with colorimetric method) and skatole (with chromatographic method), in samples of biological liquids (urine).

\section{Scientific rationale of ozone therapy}

The use of ozone in medicine is dictated by two general considerations; one based on the fact that ozone has direct and indirect effects on metabolism, and the other on the fact of its biological effects. ${ }^{6-18}$

\section{Effects of ozone on metabolisms}

- Acceleration of the use of glucose by cells for increased glycolysis, which increases the availability of ATP in the cells and therefore in the tissues, especially nerve related tissue.

- Intervention in protein metabolism due to its affinity with sulfhydryl groups, thus reacting with essential amino acids such as methionine and tryptophan or with cysteine containing sulfur.

- Direct reaction with unsaturated fatty acids, which are transformed into water-soluble compounds.

\section{Biological effects of ozone}

- In topical application there is disinfection and direct trophic action.

- For the formation of peroxides, there is a systematic antibacterial and antiviral effect. The antiseptic mechanism is similar to the one that the organism usually uses with the formation, by the leukocytes used for bacterial phagocytosis, of an antioxidant molecule, similar to that of $\mathrm{O}_{3}$, that is $\mathrm{H}_{2} \mathrm{O}_{2}$. The germicidal effect of ozone depends, above all, on the presence of water and low temperatures. It also has a great capacity to inac- tivate viruses (virustatic action) making them unable to adhere with the cell receptors on the target cell and therefore to replicate. If ozone has a virustatic action for viruses, in relation to bacteria and in particular the gram ${ }^{+}$presents a bactericidal and above all direct action.

- At the level of red blood cells, there is an increase in deformability, reducing the global blood viscosity and increase of 2,3diphosphoglycerate (responsible for the transfer of $\mathrm{O}_{2}$ from hemoglobin to tissues), which has, as a final purpose, a marked improvement in $\mathrm{O}_{2}$ transport and therefore a rheological action.

\section{Effects of peroxide products from ozone in phagocytosis}

During chronic infections, normal defensive processes are no longer able to destroy bacteria due to insufficient formation of $\mathrm{H}_{2} \mathrm{O}_{2}$. It is at this level that the positive influence of peroxides formed during ozone treatment is highlighted.

\section{Influence of ozone in the metabolism of erythrocytes}

Peroxide formation facilitates a direct activation of erythrocyte metabolism. The first step of the reaction consists in the interaction of ozone with the double bonds of unsaturated fatty acids of the phospholipid layer in the erythrocyte membrane. With the intervention of the glutathione system, an activation of glycolysis takes place, which determines an increase of 2,3-diphosphoglycerate, with relevant ease of release of $\mathrm{O}_{2}$ in tissues by the hemoglobin.

\section{Materials and Methods}

The study was conducted on an adult population from January 2012 to June 2013. 34 individuals were considered, regardless of age and sex, of which 23 females (67.6\%) and 11 males $(32.4 \%)$, aged between 27 and 62 (average: 43.9 years).

The hyperozonated water, produced by means of the OM3 ozone generator (of the company, Multiossigen) was administered in the quantity of three (3) $125 \mathrm{ml}$ glasses each per day.

Rectal insufflation was carried out 3 times a week; the ozone concentration (produced with the Multiossigen Medical 95 device) was $40 \mu \mathrm{g} / \mathrm{ml}$, while the total amount of the $\mathrm{O}_{2}-\mathrm{O}_{3}$ mixture was $250 \mathrm{cc}$ per session, the total ozone concentration administered was $10,000 \mu \mathrm{g}$.

All patients were adequately informed on the use of the treatment method for the control of a few classic symptoms of intestinal dysbiosis and expressed their consent to this type of therapy.

For admission to treatment, adult patients were examined, who had to meet the following inclusion criteria: adult males and females (age: 18-70 years) with intestinal dysbiosis, not severe, confirmed with clinical and instrumental examination, capable of giving consent to the study after adequate information, able to guarantee sufficient adherence to the prescribed therapy and motivated to complete the study.

\section{Patient assessment prior to therapy}

All patients underwent an initial descriptive pain assessment and an algometric measurement of the pain threshold, to exclude subjects with evident chronic pain, through:

\section{Algometric threshold with sphygmomanometer on the dominant upper limb}

The algometric threshold with the sphygmomanometer was recorded using the following procedure:

- The patient lies down on the examination table, with the clothing of the lower body removed. 
- The patient is told to relax keeping the upper extremities still, and the cuff of the sphygmomanometer is wrapped around the muscles of the dominant arm (triceps and biceps); the patient is instructed to report when, by inflating the sphygmomanometer, he/she senses pressure that turns into pain, i.e. when it begins to assume the characteristic of pain.

- The device is pumped every 5 seconds, generating enough air to increase the pressure by $30 \mathrm{mmHg}$ each time.

- About fifteen strokes are carried out: the first 6-8 in rapid succession in order to get to $150 \mathrm{mmHg}$ and then at a slower pace until reaching $300 \mathrm{mmHg}$, obviously if the patient does not feel pain before reaching that level.

- When and if the patient feels pain, the operation is interrupted and the pressure value at which this occurred is recorded in the file.

\section{Post-ischemic stasis test}

- With the patient again lying down, the test is carried out on the dominant arm.

- The patient is informed on what he/she may feel and instructed not to move any muscle of the upper limb.

- After positioning the sphygmomanometer on the arm, the patient's hand is grasped, holding the arm upwards, relaxed, in order to let the blood flow.

- The sphygmomanometer's cuff is insufflated up to a 30-40 $\mathrm{mmHg}$ pressure higher than the systolic, until no more blood goes to the arm.

- The arm is positioned horizontally on the examination table and, without any movement, two minutes are left to pass; in the meantime, the patient is asked if he/she feels pain. This, however, should not result in any sensation other than pins and needles.

- After two minutes of ischemia, the sphygmomanometer's cuff is deflated to about $80-90 \mathrm{mmHg}$ to create stasis and the stopwatch is activated, and the patient is requested to say something when he/she feels pain; normally he/she should not feel pain before 1 minute: i) pain at 1 minute or more $=$ normal threshold; ii) pain between 30 seconds and 1 minute = moderately low threshold; iii) pain before 30 seconds $=$ very low threshold.

For the evaluation of abdominal pressure-like pain and discomfort caused by other typical disorders of dysbiosis, a visualanalogue scale (VAS) was chosen, according to the Scott and Huskisson model, considered as a subjective measure with a single dimension, as it only evaluates one component of pain at a time.

The visual analogue scale is the visual representation of the scope of pain that a patient believes to perceive. The scope may assume different forms, both as a pain scale and as a pain relief scale.
The answers to the questionnaire may appear to be influenced by factors that affect the patient's psychophysical conditions.

The optimal length for measuring the pain appears to be $10 \mathrm{~cm}$. One extreme indicates the absence of pain, while the other represents the worst pain imaginable (Figure 1).

The scale was compiled by patients who were asked to indicate with a mark on the line, to represent the level of pain experienced. The distance is measured in numerical value, starting from the end indicating the absence of pain, to represent the extent of the pain felt, at that moment, by the patient.

VAS scale was proposed to patients undergoing oxygen and ozone mixture treatment at the beginning of recruitment (before treatment), after 45 days and at the end of the treatment after 90 days, asking them to mark, on the line between the two extremes, the extent of the pain or symptoms taken into consideration, compared to the previous assessment.

The fundamental criterion for evaluating treatment efficacy was the percentage of patients who, at the end of the treatment, showed a significant reduction in the intensity of the symptoms taken into consideration, equal to at least $10 \%$ of the baseline value.

The characteristics of the individuals treated are summarized in Figures 2-5 in time 0, at 45 and 90 days, at the end of the treatment.

The data, certainly interesting, reflected an apparent and notable improvement from a statistical point of view regarding constipation, meteorism, abdominal pains, gastralgia and chronic asthenia, assessed through the VAS, according to the Scott and Huskisson model, in individuals treated with a $\mathrm{O}_{2}-\mathrm{O}_{3}$ mixture.

\section{Results and Discussion}

All individuals presented a significant improvement, already by the first assessment, which became considerably more significant, from a statistical point of view, by the end of the study.

The results of the study made it possible to verify - first of all - that intestinal dysbiosis is a disease with a higher incidence with respect to what clinical data does not allow to establish on the basis of subjective and objective symptoms.

On the basis of the obtained results, in terms of improvement of the parameters considered, and the related symptoms, the rectal insufflation association of the oxygen-ozone mixture with the intake of hyperozonated water by mouth, has recorded quite

\section{SCALA ANALOGICA VISIVA o VAS}

( analogo visivo di Scott - Huskisson )

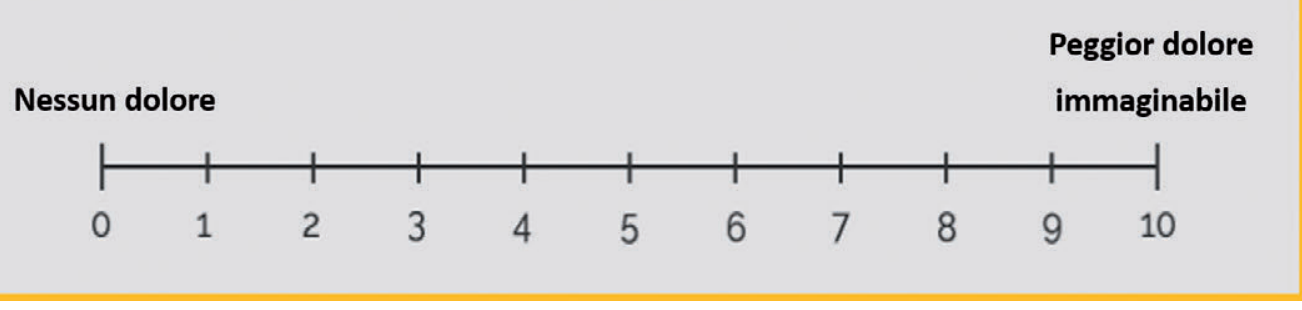

Figure 1. Visual analogue scale. 
encouraging results to such an extent that the authors have concluded that this synergy of approach can be considered as a fundamental method in the treatment of intestinal dysbiosis in those patients resistant to other treatments.

Considering that intestinal dysbiosis is a true pathology potentially involving the entire Regulatory System, forming the basis for the multifactorial pathogenesis of many other diseases, especially of a chronic nature (allergies, immune deficiency diseases, rheumatic diseases, cardiovascular diseases, headaches, neuroses, etc.), it seems particularly important to carry out a diagnostic evaluation by assessing the indole and skatole in urine, for both a preventive purpose, in individuals with apparently good health, and for therapeutic purposes, in patients suffering from chronic diseases of an allergic and/or degenerative

\section{Totale soggetti ( T0-T45 )}

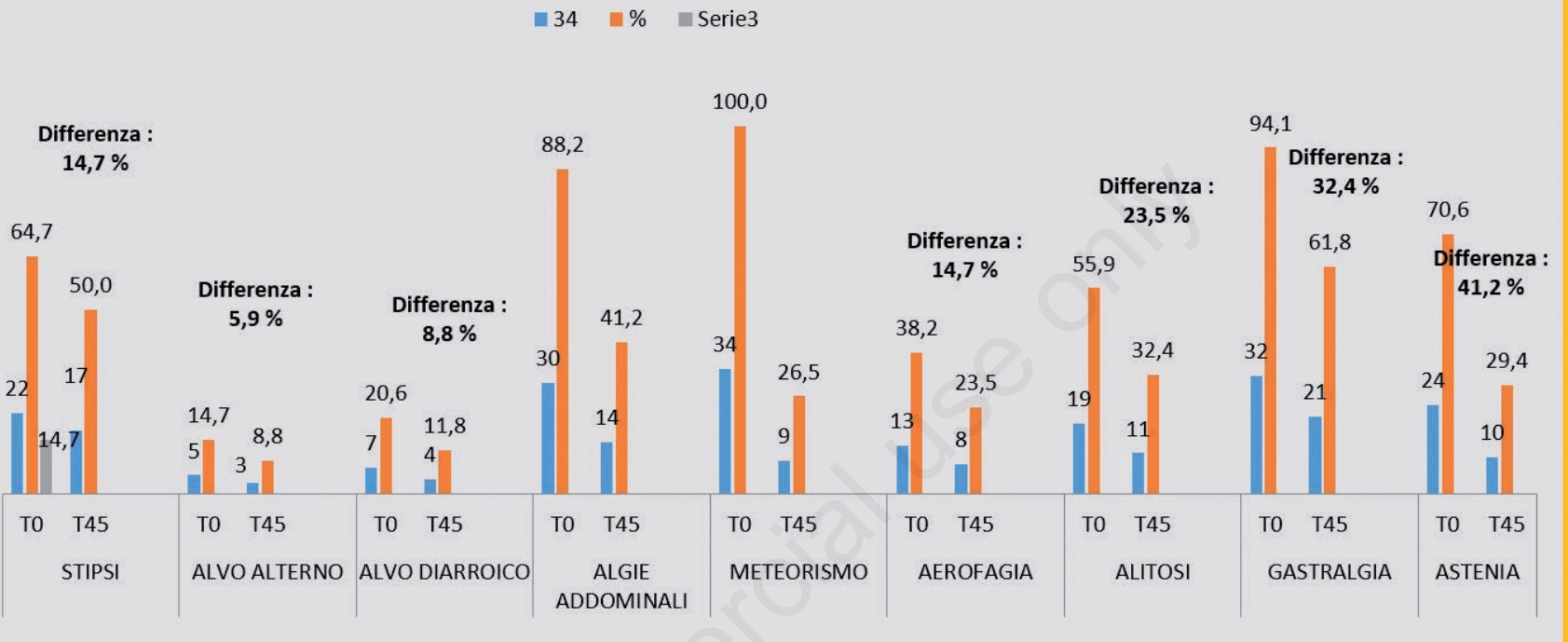

Figure 2. Characteristics of the individuals treated for constipation, meteorism and abdominal pains in time 0 , at 45 days, at the end of the treatment.

\section{Totale soggetti ( T0-T45 )}

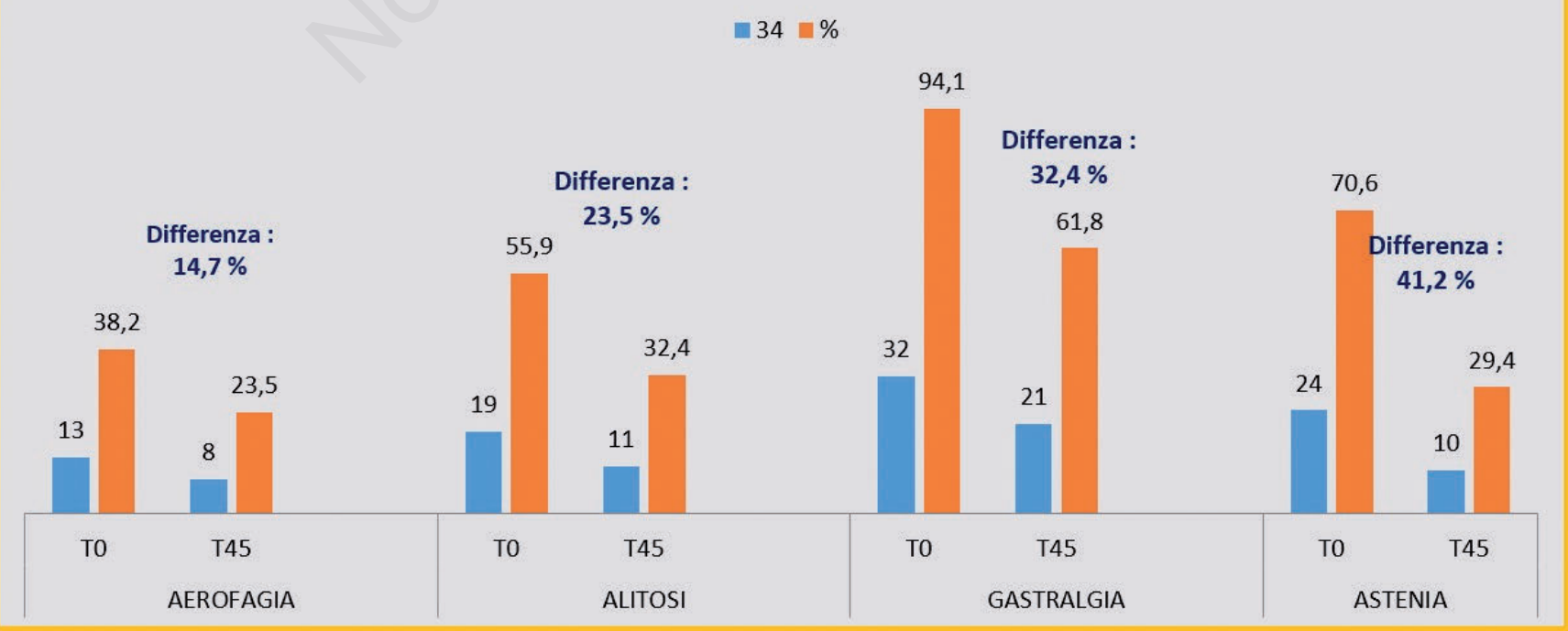

Figure 3. Characteristics of the individuals treated for gastralgia and chronic asthenia time 0 , at 45 days, at the end of the treatment. 
nature, caused or aggravated by intestinal dysbiosis. This observational study may be, therefore, a starting point for the development of new intervention strategies based on the use of oxygen and ozone mixture in view of a general improvement of health conditions in a modern western society, increasingly afflicted by this pathology that is only the tip of the iceberg of a long series of symptoms and ailments afflicting it, as it is based on a dietary lifestyle that is anything but healthy.

\section{Conclusions}

In conclusion, the study confirmed the validity of the treatment with ozonized water combined with rectal insufflation of oxygen and ozone mixture, to control the symptoms linked to dysbiosis and to favor the restoration of proper environmental homeostasis at an intestinal microclimate level in adults.

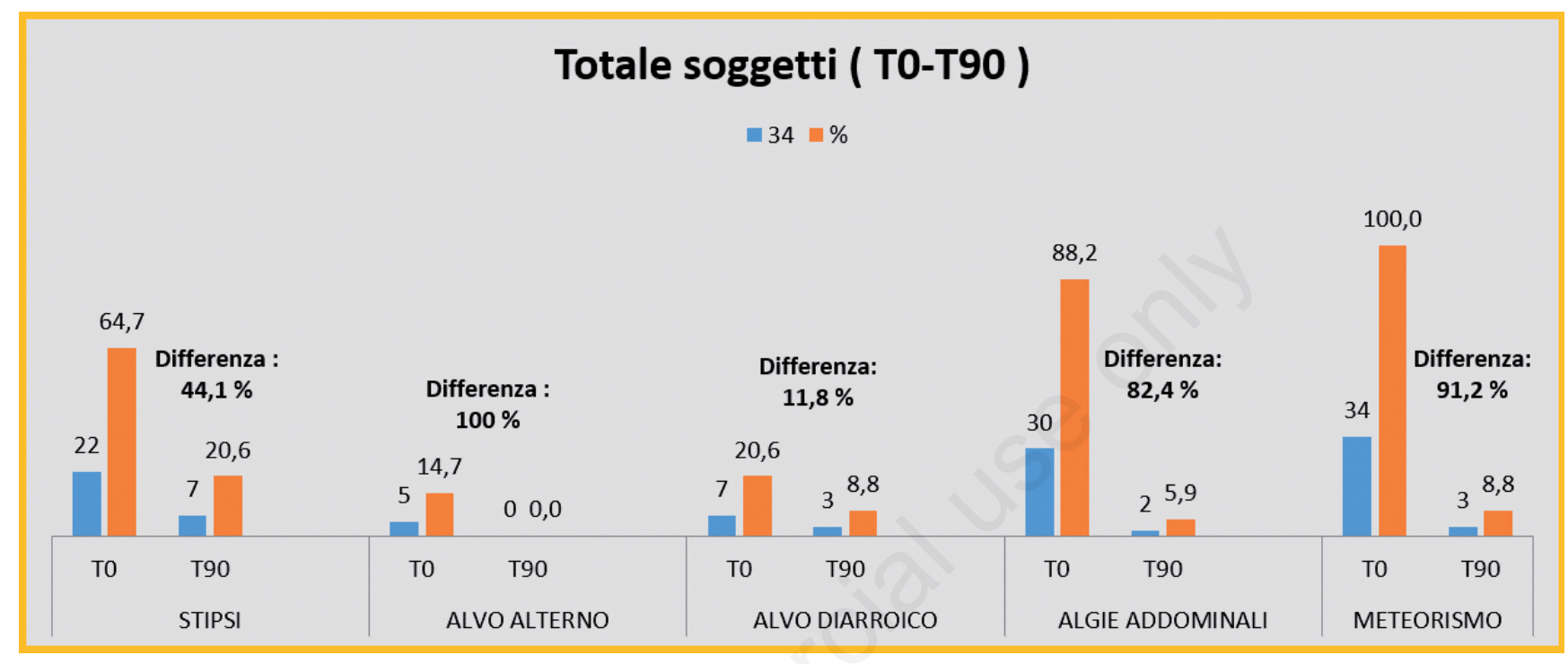

Figure 4. Characteristics of the individuals treated for constipation, meteorism and abdominal pains in time 0 , at 90 days, at the end of the treatment.

\section{Totale soggetti ( TO-T90 )}

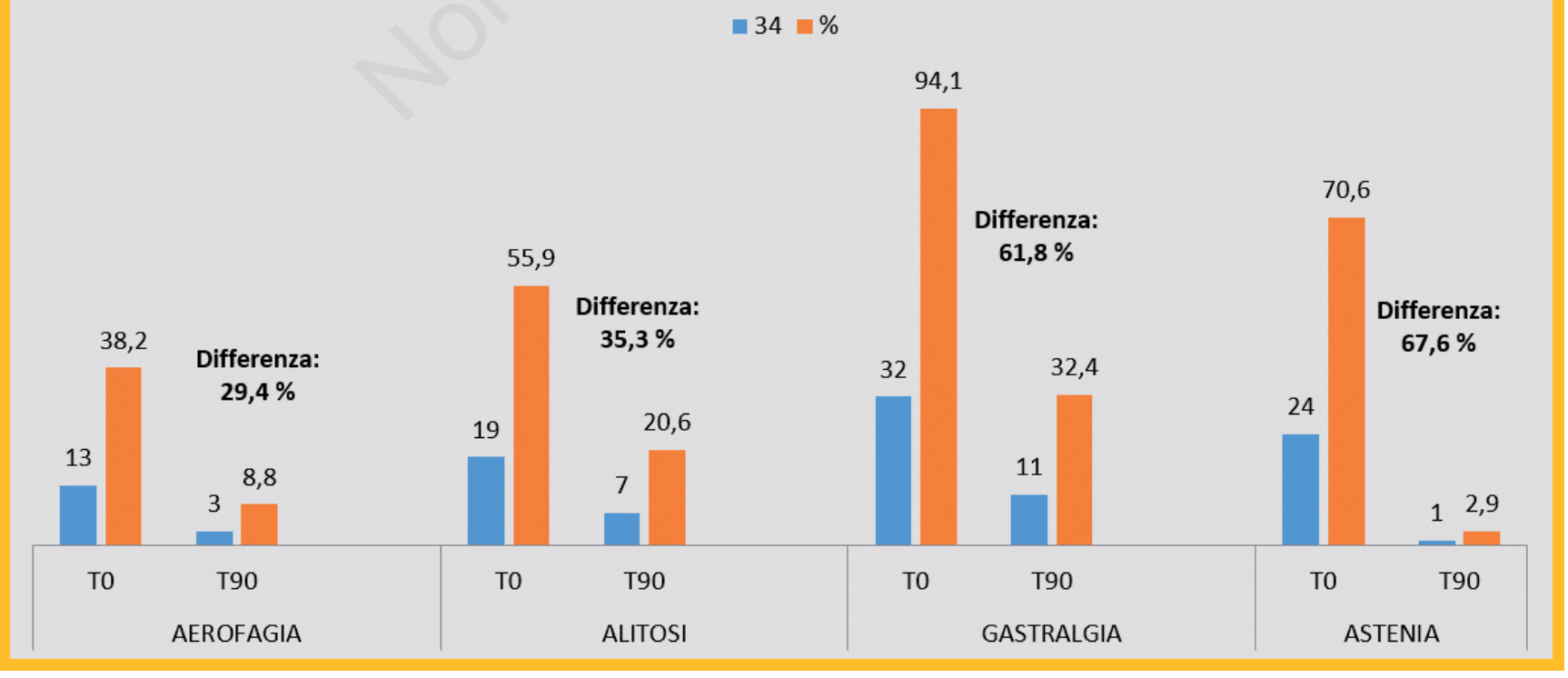

Figure 5. Characteristics of the individuals treated for gastralgia and chronic asthenia time 0 , at 90 days, at the end of the treatment. 


\section{References}

1. Dethlefsen L, Fall-Ngai M, Relman DA. An ecological and evolutionary perspective on human-microbe mutualism and disease. Nature 2007;449:811-8.

2. Lin CS, Chang CJ, Lu CC, et al. Impact of the gut microbiota, prebiotics, and probiotics on human health and disease. Biomed J 2014;37:259-68.

3. Reid G, Sanders ME, Gaskins HR, et al. New scientific paradigms for probiotics and prebiotics. J Clin Gastroenterol 2003; 37:105-18.EARCH

4. Schippa S, Conte MP. Dysbiotic events in gut microbiota: impact on human health. Nutrients 2014;6:5786-805.

5. Mearin F, Rey E, Balboa A. Motility and functional gastrointestinal disorders Gastroenterol Hepatol 2014;37 Suppl 3:3-13.

6. Franzini M. Lezioni presso il Master di $2^{\circ}$ livello in Ossigeno/Ozonoterapia. Pavia: Università di Pavia.

7. Loprete F. Lezioni presso il Master di $2^{\circ}$ livello in Ossigeno/ Ozonoterapia. Pavia: Università di Pavia.

8. Rodolico V, Tomasello G. Hsp60 and Hsp10 increase in colon mucosa of Crohn's disease and ulcerative colitis. Cell Stress Chaperones 2010;15:877-84.

9. Montalto M, Santoro L, Curigliano V, et al. Faecal calprotectin concentrations in untreated coeliac patients. Scand J Gastroenterol 2007;42:957-61.
10. Vaiano F, Loprete F. Large auto-hemoinfusion versus rectal insufflation in patients with metabolic syndrome. Gorle (BG): Oxygen-Ozone Therapy Scientific Society.

11. Bocci V, Zanardi I, Huijberts MS, Travagli V. Diabetes and chronic oxidative stress. A perspective based on the possible usefulness of ozone therapy. Siena: University of Siena.

12. Bocci V, Aldinucci C. Biochemical modifications induced in human blood by oxygenation-ozonation. J Biochem Mol Toxicol 2006;20:133-8.

13. Bocci V, Luzzi E, Corradeschi F, et al. Studies on the biological effects of ozone, 3 : an attempt to define conditions for optimal induction of cytokines. Lymph Cytok Res 1993;12:121-6.

14. Shanahan F. Probiotics and inflammatory bowel disease: is there a scientific rationale. Inflamm Bowel Dis 2000;6:107-15.

15. Porcellini A, Dall'Aglio R, Ubaldi A. Studies on the biological effects of ozone: induction of tumor necrosis factor (TNF-) on human leucocytes. Cremona: Dipartimento di Ematologia; 1994.

16. Arthur JC, Perez-Chanona E, Muhlbauer M, et al. Intestinal inflammation targets cancer-inducing activity of the microbiota. Science 2012;338:120-3.

17. Iliev ID, Funari VA, Taylor KD, et al. Interactions between commensal fungi and the C-type lectin receptor dectin-1 influence colitis. Science 2012;336:1314-7.

18. Handley SA, Thackray LB, Zhao G, et al. Pathogenic simian immunodeficiency virus infection is associated with expansion of the enteric virome. Cell 2012;151:253-66. 